\title{
Syntheses, X-ray structures, and reactions of ruthenium carbonyl complexes containing 1,1-dithiolates
}

\author{
Kom-Bei Shiu ${ }^{\text {a,* }}$, Shin-Jay Yu ${ }^{\text {a }}$, Yu Wang ${ }^{\text {b }}$, Gene-Hsiang Lee ${ }^{\mathrm{b}}$ \\ a Department of Chemistry, National Cheng Kung University, Tainan 701, Taiwan, ROC \\ ${ }^{\mathrm{b}}$ Instrument Center, National Taiwan University, Taipei 106, Taiwan, ROC
}

Received 27 September 2001; received in revised form 15 November 2001; accepted 26 November 2001

\begin{abstract}
Treatment of $\left[\mathrm{Ru}_{2}(\mathrm{CO})_{4}(\mathrm{MeCN})_{6}\right]\left[\mathrm{BF}_{4}\right]_{2}$ or $\left[\mathrm{Ru}_{2}(\mathrm{CO})_{4}\left(\mu-\mathrm{O}_{2} \mathrm{CMe}\right)_{2}(\mathrm{MeCN})_{2}\right]$ with uni-negative 1,1 -dithiolate anions via potassium dimethyldithiocarbamate, sodium diethyldithiocarbamate, potassium tert-butylthioxanthate, and ammonium $O, O^{\prime}$-diethylthiophosphate gives both monomeric and dimeric products of $c i s-\left[\mathrm{Ru}(\mathrm{CO})_{2}\left(\eta^{2}-(\mathrm{SS})\right)_{2}\right]\left((\mathrm{SS})^{-}=\mathrm{Me}_{2} \mathrm{NCS}_{2}^{-}(\mathbf{1}), \mathrm{Et}_{2} \mathrm{NCS}_{2}^{-}(\mathbf{2})\right.$, ${ }^{t} \mathrm{BuSCS}_{2}^{-}$(3), (EtO) $)_{2} \mathrm{PS}_{2}^{-}$(4)) and $\left[\mathrm{Ru}(\mathrm{CO})\left(\eta^{2}-\left(\mathrm{Me}_{2} \mathrm{NCS}_{2}\right)\right)\left(\mu, \eta^{2}-\mathrm{Me}_{2} \mathrm{NCS}_{2}\right)\right]_{2}$ (5). The lightly stabilized $\mathrm{MeCN}$ ligands of $\left[\mathrm{Ru}_{2}(\mathrm{CO})_{4}(\mathrm{MeCN})_{6}\right]\left[\mathrm{BF}_{4}\right]_{2}$ are replaced more readily than the bound acetate ligands of $\left[\mathrm{Ru}_{2}(\mathrm{CO})_{4}\left(\mu-\mathrm{O}_{2} \mathrm{CMe}\right)_{2}(\mathrm{MeCN})_{2}\right]$ by thiolates to produce $c i s-\left[\mathrm{Ru}(\mathrm{CO})_{2}\left(\eta^{2}-(\mathrm{SS})\right)_{2}\right]$ with less selectivity. Structures $\mathbf{1}$ and $\mathbf{5}$ were determined by X-ray crystallography. Although the two chelating dithiolates are cis to each other in 1, the dithiolates are trans to each other in each of the $\left\{\mathrm{Ru}(\mathrm{CO})\left(\eta^{2}-\mathrm{Me}_{2} \mathrm{NCS}_{2}\right)_{2}\right\}$ fragment of $\mathbf{5}$. The dimeric product 5 can be prepared alternatively from the decarbonylation reaction of 1 with a suitable amount of $\mathrm{Me}_{3} \mathrm{NO}$ in $\mathrm{MeCN}$. However, the dimer $\left[\mathrm{Ru}(\mathrm{CO})\left(\eta^{2}-\mathrm{Et}_{2} \mathrm{NCS}_{2}\right)\left(\mu, \eta^{2}-\mathrm{Et}_{2} \mathrm{NCS}_{2}\right)\right]_{2}(\mathbf{6})$, prepared from the reaction of 2 with $\mathrm{Me}_{3} \mathrm{NO}$, has a structure different from 5. The spectral data of $\mathbf{6}$ probably indicate that the two chelating dithiolates are cis to each other in one $\left\{\mathrm{Ru}(\mathrm{CO})\left(\eta^{2}-\mathrm{Et}_{2} \mathrm{NCS}_{2}\right)_{2}\right\}$ fragment but trans in the other. Both 5 and $\mathbf{6}$ react readily at ambient temperature with benzyl isocyanide to yield cis-[Ru(CO) $\left.\left(\mathrm{CNCH}_{2} \mathrm{Ph}\right)\left(\eta^{2}-(\mathrm{SS})\right)_{2}\right]\left(\left(\mathrm{SS}_{)}=\mathrm{Me}_{2} \mathrm{NCS}_{2}^{-}(7)\right.\right.$ and $\left.\mathrm{Et}_{2} \mathrm{NCS}_{2}^{-}(\mathbf{8})\right)$. A dimerization pathway for $c i s-\left[\mathrm{Ru}(\mathrm{CO})_{2}\left(\eta^{2}-(\mathrm{SS})\right)_{2}\right]$ via decabonylation and isomerization is proposed. (C) 2002 Elsevier Science B.V. All rights reserved.
\end{abstract}

Keywords: Ruthenium; Carbonyl; Dithiocarbamate; Thioxanthate; Dithiophosphate; Alkyl isocyanide

\section{Introduction}

In the course of a program of synthesis and structural characterization of a series of 1,1-dithiolate complexes of the type cis-[Ru(CO) $\left.)_{2}\left(\eta^{2}-(\mathrm{SS})\right)_{2}\right]\left((\mathrm{SS})^{-}=\right.$ $\mathrm{Me}_{2} \mathrm{NCS}_{2}^{-}$(1), $\mathrm{Et}_{2} \mathrm{NCS}_{2}^{-}$(2), ${ }^{t} \mathrm{BuSCS}_{2}^{-}$(3), (EtO) ${ }_{2} \mathrm{PS}_{2}^{-}$ (4)), following a similar procedure reported for cis$\left[\mathrm{Ru}(\mathrm{CO})_{2}\left(\eta^{2}-\mathrm{S}_{2} \mathrm{PMe}_{2}\right)_{2}\right]$ from $\left[\mathrm{Ru}_{2}(\mathrm{CO})_{4}\left(\mu-\mathrm{O}_{2} \mathrm{CMe}\right)_{2}-\right.$ $(\mathrm{MeCN})_{2}$ ] [1], we have obtained on one occasion small amount of another product (5), either from $\left[\mathrm{Ru}_{2}(\mathrm{CO})_{4}\left(\mu-\mathrm{O}_{2} \mathrm{CMe}\right)_{2}(\mathrm{MeCN})_{2}\right]$ or a derived complex with lightly stabilized ligands $\mathrm{MeCN}, \quad\left[\mathrm{Ru}_{2}(\mathrm{CO})_{4^{-}}\right.$ $\left.(\mathrm{MeCN})_{6}\right]\left[\mathrm{BF}_{4}\right]_{2}$ [2]. The elemental analysis results of 5 appear consistent with the formulation of $\left[\mathrm{Ru}(\mathrm{CO})\left(\eta^{2}-\right.\right.$

\footnotetext{
* Corresponding author. Fax: + 886-6-274-0552.

E-mail address: kbshiu@mail.ncku.edu.tw (K.-B. Shiu).
}

$\left.\left.\left(\mathrm{Me}_{2} \mathrm{NCS}_{2}\right)\right)\left(\mu, \eta^{2}-\mathrm{Me}_{2} \mathrm{NCS}_{2}\right)\right] \cdot 1 / 2 \mathrm{CH}_{2} \mathrm{Cl}_{2}$, but the real structure cannot be assigned without any ambiguity. It may be a mononuclear product with a half $\mathrm{CH}_{2} \mathrm{Cl}_{2}$ molecule as one chloro ligand around $\mathrm{Ru}$ [3], or a dinuclear product, as a $\mathrm{CH}_{2} \mathrm{Cl}_{2}$ solvate, through sulfur coordination in a chelating-bridging mode [4]. If it is a dimeric compound, the related structure may adopt one of the three possible configurations: configuration $\mathbf{A}$ contains two cis-disposed dithiolates; configuration $\mathbf{B}$ contains two trans-disposed dithiolates; and configuration $\mathbf{C}$ contains one cis- and one trans-dithiolates around each metal atom (Chart 1).

We report here the results of an X-ray study of 5 which definitely settles the question of its solid-state structure to be dimeric with configuration B. This work, along with the results of an X-ray study of $\mathbf{1}$ and a reactivity study of $\mathbf{1}$ and $\mathbf{2}$, has revealed the dimerization pathway for $c i s-\left[\mathrm{Ru}(\mathrm{CO})_{2}\left(\eta^{2}-(\mathrm{SS})\right)_{2}\right]$ via decarbonylation and isomerization. 


\section{Experimental}

The compounds $\left[\mathrm{Ru}_{2}(\mathrm{CO})_{4}(\mathrm{MeCN})_{6}\right]\left[\mathrm{BF}_{4}\right]_{2}$ [2a] and $\left[\mathrm{Ru}_{2}(\mathrm{CO})_{4}\left(\mu-\mathrm{O}_{2} \mathrm{CMe}\right)_{2}(\mathrm{MeCN})_{2}\right]$ [5] were prepared according to literature methods. All the reactions were performed under prepurified nitrogen using freshly distilled solvents. ${ }^{1} \mathrm{H}$ - and ${ }^{31} \mathrm{P}-\mathrm{NMR}$ spectra were recorded in a Bruker AMC400 spectrometer $\left({ }^{1} \mathrm{H}, 400 \mathrm{MHz} ;{ }^{31} \mathrm{P}\right.$, $162 \mathrm{MHz})$ calibrated against internal deuterated solvents $\left({ }^{1} \mathrm{H}\right)$ or external $85 \% \mathrm{H}_{3} \mathrm{PO}_{4}\left({ }^{31} \mathrm{P}\right)$. IR spectra were recorded in a Bio-Rad FTS 175 instrument. Microanalyses were carried out by the staff of the Microanalytical Service of the Department of Chemistry, National Cheng Kung University.

\subsection{Preparation of cis- $\left[\mathrm{Ru}(\mathrm{CO})_{2}\left(\eta^{2}-\mathrm{Me}_{2} \mathrm{NCS}_{2}\right)_{2}\right]$ (1) and $\left[\mathrm{Ru}(\mathrm{CO})\left(\eta^{2}-\mathrm{Me}_{2} \mathrm{NCS}_{2}\right)\left(\mu, \eta^{2}-\mathrm{Me}_{2} \mathrm{NCS}_{2}\right)\right]_{2}$ (5)}

\subsubsection{Method A}

Potassium dimethyldithiocarbamate monohydrate $(0.830 \mathrm{~g}, 4.68 \mathrm{mmol})$ was added directly to a stirred yellow solution of $\left[\mathrm{Ru}_{2}(\mathrm{CO})_{4}\left(\mu-\mathrm{O}_{2} \mathrm{CMe}\right)_{2}(\mathrm{MeCN})_{2}\right]$, prepared in situ from catena- $\left[\mathrm{Ru}(\mathrm{CO})_{2}\left(\mu, \eta^{2}-\mathrm{O}_{2} \mathrm{CMe}\right)\right]$ $(0.190 \mathrm{~g}, 0.88 \mathrm{mmol})$, in $36 \mathrm{ml}$ of THF. The color changed immediately to orange-brown. The mixture was stirred for $3.5 \mathrm{~h}$ and then taken to dryness under vacuum. Recrystallization using $\mathrm{Et}_{2} \mathrm{O}-\mathrm{MeOH}$ from this solid residue gave $0.244 \mathrm{~g}$ pale-yellow cis- $\left[\mathrm{Ru}(\mathrm{CO})_{2}\left(\eta^{2}-\right.\right.$ $\left.\left.\mathrm{Me}_{2} \mathrm{NCS}_{2}\right)_{2}\right]$ (1) (yield 70\%). Then, recrystallization using $\mathrm{CH}_{2} \mathrm{Cl}_{2}$-hexane from the remaining solid gave orange-brown $\quad\left[\mathrm{Ru}(\mathrm{CO})\left(\eta^{2}-\mathrm{Me}_{2} \mathrm{NCS}_{2}\right)\left(\mu, \eta^{2}-\mathrm{Me}_{2}-\right.\right.$ $\left.\left.\mathrm{NCS}_{2}\right)\right]_{2} \cdot \mathrm{CH}_{2} \mathrm{Cl}_{2}$ (5) (31 mg, 9\%). 1, Anal. Calc. for $\mathrm{C}_{8} \mathrm{H}_{12} \mathrm{~N}_{2} \mathrm{O}_{2} \mathrm{RuS}_{4}$ : C, 24.17; H, 3.04; N, 7.05. Found: C, 23.88; H, 3.05; N, 7.03\%. ${ }^{1} \mathrm{H}-\mathrm{NMR}\left(\mathrm{CDCl}_{3}\right): \delta 3.26(\mathrm{~s}$, $6 \mathrm{H}, \mathrm{Me}), 3.28$ (s, 6H, Me). IR $\left(\mathrm{CH}_{2} \mathrm{Cl}_{2}, \mathrm{~cm}^{-1}\right): v_{\mathrm{CO}}$ 2039 (s), 1972 (s). IR (KBr, cm $\left.{ }^{-1}\right): v_{\mathrm{CO}} 2024$ (s), 1962 (s). 5, Anal. Calc. for $\mathrm{C}_{14} \mathrm{H}_{24} \mathrm{~N}_{4} \mathrm{O}_{2} \mathrm{Ru}_{2} \mathrm{~S}_{8} \mathrm{CH}_{2} \mathrm{Cl}_{2}$ : C, 21.87; H, 3.18; N, 6.80. Found: C, 21.67; H, 3.19; N, $6.78 \%$. ${ }^{1} \mathrm{H}-\mathrm{NMR}\left(\mathrm{CDCl}_{3}\right): \delta 3.22(\mathrm{br}, 6 \mathrm{H}, \mathrm{Me}), 3.36$ (br, 6H, Me), 3.60 (br, 6H, Me), 3.62 (br, 6H, Me), 5.32 (s, $\left.2 \mathrm{H}, \mathrm{CH}_{2} \mathrm{Cl}_{2}\right)$. IR $\left(\mathrm{CH}_{2} \mathrm{Cl}_{2}, \mathrm{~cm}^{-1}\right): v_{\mathrm{CO}} 1927$ (s). IR $\left(\mathrm{KBr}, \mathrm{cm}^{-1}\right)$ : $v_{\mathrm{CO}}, 1921$ (s).

\subsubsection{Method B}

Potassium dimethyldithiocarbamate monohydrate $(0.240 \mathrm{~g}, 1.35 \mathrm{mmol})$ was added directly to a stirred orange solution of $\left[\mathrm{Ru}_{2}(\mathrm{CO})_{4}(\mathrm{MeCN})_{6}\right]\left[\mathrm{BF}_{4}\right]_{2}(0.102 \mathrm{~g}$, $0.139 \mathrm{mmol}$ ) in $20 \mathrm{ml}$ of $\mathrm{CH}_{2} \mathrm{Cl}_{2}$ and $1 \mathrm{ml}$ of $\mathrm{MeOH}$. The mixture was stirred for $2 \mathrm{~h}$ and the solvents were removed under vacuum. A procedure similar to that described above was applied, giving $76 \mathrm{mg}$ of $\mathbf{1}$ (yield $68 \%$ ) and $12 \mathrm{mg}$ of 5 (yield $11 \%$ ).

\subsection{Preparation of cis-[Ru(CO) $\left.)_{2}\left(\eta^{2}-E t_{2} \mathrm{NCS}_{2}\right)_{2}\right]$ (2)}

The yellow 2 was obtained as the only product from the reaction of $\left[\mathrm{Ru}_{2}(\mathrm{CO})_{4}(\mathrm{MeCN})_{6}\right]\left[\mathrm{BF}_{4}\right]_{2}$ with sodium diethyldithiocarbamate trihydrate by method $\mathrm{B}$. Yield: $73 \%$. Anal. Calc. for $\mathrm{C}_{12} \mathrm{H}_{20} \mathrm{~N}_{2} \mathrm{O}_{2} \mathrm{RuS}_{4}$ : C, 31.77; H, 4.44; N, 6.18. Found: C, 31.68; H, 4.45; N, 6.17\%. ${ }^{1} \mathrm{H}-\mathrm{NMR}$ (acetone- $\left.d_{6}\right): \quad \delta \quad 1.25 \quad\left(\mathrm{t}, \quad 6 \mathrm{H}, \quad \mathrm{CH}_{3} \mathrm{CH}_{2}\right.$, $\left.{ }^{3} J(\mathrm{HH})=7.2 \mathrm{~Hz}\right), 1.26\left(\mathrm{t}, 6 \mathrm{H}, \mathrm{CH}_{3} \mathrm{CH}_{2},{ }^{3} J_{\mathrm{H}, \mathrm{H}}=7.2\right.$ $\mathrm{Hz}), 3.76$ (q, $\left.4 \mathrm{H}, \mathrm{CH}_{3} \mathrm{CH}_{2},{ }^{3} J_{\mathrm{H}, \mathrm{H}}=7.2 \mathrm{~Hz}\right), 3.80$ (q, $\left.4 \mathrm{H}, \mathrm{CH}_{3} \mathrm{CH}_{2},{ }^{3} J_{\mathrm{H}, \mathrm{H}}=7.2 \mathrm{~Hz}\right)$. IR $\left(\mathrm{CH}_{2} \mathrm{Cl}_{2}, \mathrm{~cm}^{-1}\right): v_{\mathrm{CO}}$ 2035 (s), 1968 (s). IR (KBr, cm ${ }^{-1}$ ): $v_{\mathrm{CO}} 2030$ (s), 1952 (s).

\subsection{Preparation of cis $-\left[\mathrm{Ru}(\mathrm{CO})_{2}\left(\eta^{2}{ }^{t} \mathrm{BuSCS}_{2}\right)_{2}\right]$ (3)}

The orange-brown 3 was obtained as the only product from the reaction of $\left[\mathrm{Ru}_{2}(\mathrm{CO})_{4}(\mathrm{MeCN})_{6}\right]\left[\mathrm{BF}_{4}\right]_{2}$ with potassium tert-butylthioxanthate by method $\mathrm{B}$. Yield: $67 \%$. Anal. Calc. for $\mathrm{C}_{12} \mathrm{H}_{18} \mathrm{O}_{2} \mathrm{RuS}_{6}$ : C, 29.55; $\mathrm{H}$, 3.72. Found: C, 29.34; H, 3.75\%. ${ }^{1} \mathrm{H}-\mathrm{NMR}$ (acetone$\left.d_{6}\right): \delta 1.71\left(\mathrm{~s}, 18 \mathrm{H},\left(\mathrm{CH}_{3}\right)_{3} \mathrm{C}\right)$. IR $\left(\mathrm{CH}_{2} \mathrm{Cl}_{2}, \mathrm{~cm}^{-1}\right): v_{\mathrm{CO}}$, 2051 (s), 1991 (s). IR (KBr, cm $\left.{ }^{-1}\right): v_{\mathrm{CO}} 2041$ (s), 1985 (s).

\subsection{Preparation of cis-[Ru(CO $\left.)_{2}\left(\eta^{2}-(E t O)_{2} P S_{2}\right)_{2}\right]$ (4)}

The orange-yellow 4 was obtained as the only product from the reaction of $\left[\mathrm{Ru}_{2}(\mathrm{CO})_{4}(\mathrm{MeCN})_{6}\right]\left[\mathrm{BF}_{4}\right]_{2}$ with ammonium $O, O^{\prime}$-diethyldithiophosphate by method B. Yield: $68 \%$ Anal. Calc. for $\mathrm{C}_{10} \mathrm{H}_{20} \mathrm{O}_{6} \mathrm{P}_{2} \mathrm{RuS}_{4}$ : C, 22.77; H, 3.82. Found: C, 22.64; $\mathrm{H}, \quad 3.87 \%$. ${ }^{1} \mathrm{H}-\mathrm{NMR}$ (acetone- $\left.d_{6}\right): \delta 1.33(\mathrm{t}, \quad 6 \mathrm{H}$, $\left.\mathrm{CH}_{3} \mathrm{CH}_{2},{ }^{3} \mathrm{~J}_{\mathrm{H}, \mathrm{H}}=7.1 \mathrm{~Hz}\right), 1.37\left(\mathrm{t}, 6 \mathrm{H}, \mathrm{CH}_{3} \mathrm{CH}_{2}\right.$, $\left.{ }^{3} J_{\mathrm{H}, \mathrm{H}}=7.0 \mathrm{~Hz}\right), 4.22\left(\mathrm{~m}, 8 \mathrm{H}, \mathrm{CH}_{3} \mathrm{CH}_{2}\right) \cdot{ }^{31} \mathrm{P}\left\{{ }^{1} \mathrm{H}\right\}-\mathrm{NMR}$ (acetone- $\left.d_{6}\right): \delta 100.8(\mathrm{~s}, 2 \mathrm{P})$. IR $\left(\mathrm{CH}_{2} \mathrm{Cl}_{2}, \mathrm{~cm}^{-1}\right): v_{\mathrm{CO}}$ 2053 (s), 1987 (s).

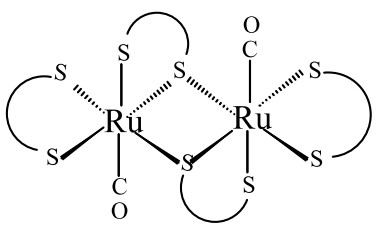

$\mathbf{A}$

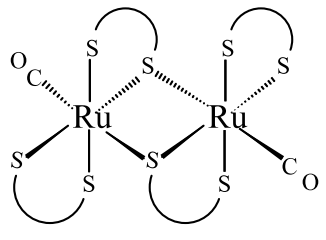

B

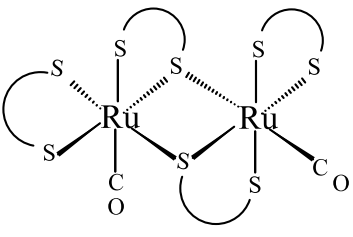

C

Chart 1. 
Table 1

Crystal data and structure refinement parameters for compounds $\mathbf{1}$ and $\mathbf{5} \cdot \mathrm{CH}_{2} \mathrm{Cl}_{2}$

\begin{tabular}{|c|c|c|}
\hline Empirical formula & $\mathrm{C}_{8} \mathrm{H}_{12} \mathrm{~N}_{2} \mathrm{O}_{2} \mathrm{Ru}_{2} \mathrm{~S}_{4}$ & $\begin{array}{l}\mathrm{C}_{15} \mathrm{H}_{26} \mathrm{Cl}_{2} \mathrm{~N}_{4} \mathrm{O}_{2-} \\
\mathrm{Ru}_{2} \mathrm{~S}_{8}\end{array}$ \\
\hline Formula weight & 397.51 & 823.92 \\
\hline Temperature (K) & $150(1)$ & $150(1)$ \\
\hline Wavelength $\left(\mathrm{Mo}-\mathrm{K}_{\alpha}\right)(\AA)$ & 0.71073 & 0.71073 \\
\hline Space group & monoclinic, $P 2_{1} / c$ & monoclinic, $C 2 / c$ \\
\hline \multicolumn{3}{|l|}{ Unit cell dimensions } \\
\hline$a(\AA)$ & $6.2803(1)$ & $10.9628(2)$ \\
\hline$b(\AA)$ & $17.6427(1)$ & $14.8235(1)$ \\
\hline$c(\AA)$ & $13.2120(1)$ & $17.0111(1)$ \\
\hline$\beta\left({ }^{\circ}\right)$ & $97.336(1)$ & $94.159(1)$ \\
\hline$V\left(\AA^{3}\right)$ & $1451.93(3)$ & $2757.14(6)$ \\
\hline$Z$ & 4 & 4 \\
\hline$D_{\text {calc }}\left(\mathrm{g} \mathrm{cm}^{-3}\right)$ & 1.818 & 1.985 \\
\hline $\mathrm{F}(000)$ & 792 & 1640 \\
\hline Crystal size (mm) & $0.30 \times 0.20 \times 0.15$ & $0.20 \times 0.10 \times 0.10$ \\
\hline $2 \theta$ Range $\left({ }^{\circ}\right)$ & $3-55$ & $3-55$ \\
\hline Index ranges $(h, k, l)$ & $\pm 8, \pm 23, \pm 17$ & $\pm 14, \pm 19, \pm 22$ \\
\hline$\mu\left(\mathrm{Mo}-\mathrm{K}_{\alpha}\right)\left(\mathrm{mm}^{-1}\right)$ & 1.645 & 1.919 \\
\hline Reflections collected & 9266 & 8254 \\
\hline $\begin{array}{l}\text { No. of observed reflections } \\
\left(N_{\mathrm{o}}\right)\end{array}$ & $3307(>2 \sigma)$ & $3121(>2 \sigma)$ \\
\hline Absorption correction & Sadabs & Sadabs \\
\hline $\mathrm{Max} / \mathrm{min}$ transmission & $0.8015 / 0.6805$ & $0.8621 / 0.7325$ \\
\hline Refinement program & NRCVAX & NRCVAX \\
\hline $\begin{array}{l}\text { No. of reflection } \\
\text { parameters }\left(N_{\mathrm{p}}\right)\end{array}$ & 155 & 147 \\
\hline$R^{\mathrm{a}}, R_{\mathrm{w}}^{\mathrm{a}}$ & $0.0255,0.0634$ & $0.0631,0.1389$ \\
\hline Goodness-of-fit a & 1.067 & 1.120 \\
\hline \multirow[t]{2}{*}{ Weighting scheme } & {$\left[\sigma^{2}\left(F_{\mathrm{o}}\right)\right.$} & {$\left[\sigma^{2}\left(F_{\mathrm{o}}\right)\right.$} \\
\hline & $\left.+0.0016 F_{\mathrm{o}}^{2}\right]^{-1}$ & $\left.+0.00029 F_{\mathrm{o}}^{2}\right]^{-1}$ \\
\hline$(\Delta \rho)_{\max }\left(\mathrm{e} \AA^{3}\right)$ & 0.505 & 2.040 \\
\hline$(\Delta \rho)_{\min }\left(\mathrm{e} \AA^{3}\right)$ & -0.502 & -1.356 \\
\hline
\end{tabular}

${ }^{\mathrm{a}} R=\left[\Sigma|| F_{\mathrm{o}}|-| F_{\mathrm{c}}|| / \Sigma\left|F_{\mathrm{o}}\right|\right] . R_{\mathrm{w}}=\left[\Sigma \omega\left(\left|F_{\mathrm{o}}\right|-\left|F_{\mathrm{c}}\right|\right)^{2} / \Sigma \omega\left|F_{\mathrm{o}}\right|^{2}\right]^{1 / 2}$. Goodness-of-fit $=\left[\Sigma \omega\left(\left|F_{\mathrm{o}}\right|-\mid F_{\mathrm{c}}\right)^{2} / N_{\mathrm{o}}-N_{\mathrm{p}}\right]^{1 / 2}$.

\subsection{Reaction between cis-[Ru(CO) $\left.)_{2}\left(\eta^{2}-\mathrm{Me}_{2} \mathrm{NCS}_{2}\right)_{2}\right]$ (1) and $\mathrm{Me}_{3} \mathrm{NO}$}

Trimethylamine $N$-oxide dihydrate $(0.072 \mathrm{~g}, 0.65$ mmol) was added directly to a stirred yellow suspension of $1(0.218 \mathrm{~g}, 0.55 \mathrm{mmol})$ in $20 \mathrm{ml}$ of $\mathrm{MeCN}$. The mixture was stirred at ambient temperature for $10 \mathrm{~h}$, and the volume of the solvent was reduced to ca. $1 \mathrm{ml}$. Twenty milliliters of $\mathrm{CH}_{2} \mathrm{Cl}_{2}$ was then added to the suspension. After filtration, the orange-brown solid was washed thoroughly with $\mathrm{CH}_{2} \mathrm{Cl}_{2}(20 \mathrm{ml})$ twice and dried under vacuum to give $\left[\mathrm{Ru}(\mathrm{CO})\left(\eta^{2}-\right.\right.$ $\left.\left.\mathrm{Me}_{2} \mathrm{NCS}_{2}\right)\left(\mu, \eta^{2}-\mathrm{Me}_{2} \mathrm{NCS}_{2}\right)\right]_{2} \cdot \mathrm{CH}_{2} \mathrm{Cl}_{2}$ (5) $(0.214 \mathrm{~g}, 95 \%)$.

\subsection{Reaction between cis-[Ru(CO) $\left.{ }_{2}\left(\eta^{2}-E t_{2} N_{C S}\right)_{2}\right]$ (2) and $\mathrm{Me}_{3} \mathrm{NO}$}

Trimethylamine $N$-oxide dihydrate $(0.119 \mathrm{~g}, 1.07$ mmol) was added directly to a stirred yellow suspension of $2(0.406 \mathrm{~g}, 0.90 \mathrm{mmol})$ in $20 \mathrm{ml}$ of $\mathrm{MeCN}$. The mixture was stirred at ambient temperature for $22 \mathrm{~h}$, and the solvent was stripped off, giving a yellowbrown solid. Recrystallization from $\mathrm{CH}_{2} \mathrm{Cl}_{2}-\mathrm{MeOH}$ gave yellow $\left[\mathrm{Ru}(\mathrm{CO})\left(\eta^{2}-\mathrm{Et}_{2} \mathrm{NCS}_{2}\right)\left(\mu, \eta^{2}-\mathrm{Et}_{2} \mathrm{NCS}_{2}\right)\right]_{2}$ (6) (0.335 g, 88\%). Anal. Calc. for $\mathrm{C}_{22} \mathrm{H}_{40} \mathrm{~N}_{4} \mathrm{O}_{2} \mathrm{Ru}_{2} \mathrm{~S}_{8}$ : C, 31.04; H, 4.74; N, 6.58. Found: C, 30.88; H, 4.73; N, 6.59\%. ${ }^{1} \mathrm{H}-\mathrm{NMR}\left(\mathrm{CD}_{2} \mathrm{Cl}_{2}\right): \delta 1.19\left(\mathrm{~m}, 9 \mathrm{H}, \mathrm{CH}_{3} \mathrm{CH}_{2}\right)$, $1.24\left(\mathrm{~m}, 9 \mathrm{H}, \mathrm{CH}_{3} \mathrm{CH}_{2}\right), 1.37\left(\mathrm{~m}, 6 \mathrm{H}, \mathrm{CH}_{3} \mathrm{CH}_{2}\right), 3.45$ $\left(\mathrm{m}, 4 \mathrm{H}, \mathrm{CH}_{3} \mathrm{CH}_{2}\right), 3.61\left(\mathrm{~m}, 2 \mathrm{H}, \mathrm{CH}_{3} \mathrm{CH}_{2}\right), 3.78(\mathrm{~m}$, $\left.6 \mathrm{H}, \mathrm{CH}_{3} \mathrm{CH}_{2}\right), 3.88\left(\mathrm{~m}, 2 \mathrm{H}, \mathrm{CH}_{3} \mathrm{CH}_{2}\right), 4.15(\mathrm{~m}, 2 \mathrm{H}$, $\left.\mathrm{CH}_{3} \mathrm{CH}_{2}\right)$. IR $\left(\mathrm{CH}_{2} \mathrm{Cl}_{2}, \mathrm{~cm}^{-1}\right): v_{\mathrm{CO}} 1925$ (s). IR ( $\mathrm{KBr}$, $\left.\mathrm{cm}^{-1}\right): v_{\mathrm{CO}} 1943(\mathrm{~s}), 1924(\mathrm{~s})$.

\subsection{Preparation of \\ cis-[Ru(CO)(CNCH $\left.\mathrm{Ch}_{2} \mathrm{P}\left(\eta^{2}-\mathrm{Me}_{2} \mathrm{NCS}_{2}\right)_{2}\right]$ (7)}

To a stirred suspension of $5(0.294 \mathrm{~g}, 0.357 \mathrm{mmol})$ in $\mathrm{CH}_{2} \mathrm{Cl}_{2}(50 \mathrm{ml}) 0.2 \mathrm{ml}$ of $\mathrm{PhCH}_{2} \mathrm{NC}$ (ca. $1.61 \mathrm{mmol}$ ) was added. The mixture was stirred at ambient temperature for 3 days, and the volatile was removed under vacuum. Recrystallization from $\mathrm{CH}_{2} \mathrm{Cl}_{2}-\mathrm{MeOH}$ gave yellow cis-[Ru(CO) $\left.\left(\mathrm{CNCH}_{2} \mathrm{Ph}\right)\left(\eta^{2}-\mathrm{Me}_{2} \mathrm{NCS}_{2}\right)_{2}\right] \quad$ (7) $(0.165 \mathrm{~g}, 95 \%)$. Anal. Calc. for $\mathrm{C}_{15} \mathrm{H}_{19} \mathrm{~N}_{3} \mathrm{ORuS}_{4}$ : C, 37.02; H, 3.93; N, 8.63. Found: C, 36.89; H, 3.94; N, $8.63 \% .{ }^{1} \mathrm{H}-\mathrm{NMR}$ (acetone- $d_{6}$ ): $\delta 3.20(\mathrm{br}, 6 \mathrm{H}, \mathrm{Me}), 3.21$ (br, 6H, Me), $7.37(\mathrm{~m}, 5 \mathrm{H}, \mathrm{Ph}), 7.51\left(\mathrm{~d}, 1 \mathrm{H}, \mathrm{CNCH}_{2} \mathrm{Ph}\right.$, $\left.{ }^{2} J_{\mathrm{H}, \mathrm{H}}=7.6 \mathrm{~Hz}\right), 7.56\left(\mathrm{~d}, 1 \mathrm{H}, \mathrm{CNCH}_{2} \mathrm{Ph},{ }^{2} J_{\mathrm{H}, \mathrm{H}}=7.6\right.$ $\mathrm{Hz})$. IR $\left(\mathrm{CH}_{2} \mathrm{Cl}_{2}, \mathrm{~cm}^{-1}\right): v_{\mathrm{CN}} 2109$ (s); $v_{\mathrm{CO}} 1962$ (s). IR $\left(\mathrm{KBr}, \mathrm{cm}^{-1}\right): v_{\mathrm{CN}} 2105(\mathrm{~s}) ; v_{\mathrm{CO}} 1971(\mathrm{~s})$.

\subsection{Preparation of cis- $\left[\mathrm{Ru}(\mathrm{CO})\left(\mathrm{CNCH}_{2} \mathrm{Ph}\right)\left(\eta^{2}-\mathrm{Et}_{2} \mathrm{NCS}_{2}\right)_{2}\right](\boldsymbol{8})$}

To a stirred solution of $6(0.136 \mathrm{~g}, 0.319 \mathrm{mmol})$ in $\mathrm{CH}_{2} \mathrm{Cl}_{2}(15 \mathrm{ml}) 0.2 \mathrm{ml}$ of $\mathrm{PhCH}_{2} \mathrm{NC}$ (ca. $1.61 \mathrm{mmol}$ ) was added. The mixture was stirred at ambient temperature for $2 \mathrm{~h}$, and the volatile was removed under vacuum. Recrystallization from $\mathrm{CH}_{2} \mathrm{Cl}_{2}$-hexane gave yellow cis-[Ru(CO) $\left.\left(\mathrm{CNCH}_{2} \mathrm{Ph}\right)\left(\eta^{2}-\mathrm{Et}_{2} \mathrm{NCS}_{2}\right)_{2}\right] \quad$ (8) (0.169 g, 97\%). Anal. Calc. for $\mathrm{C}_{19} \mathrm{H}_{27} \mathrm{~N}_{3} \mathrm{ORuS}_{4}$ : C, 42.04; H, 5.01; N, 7.74. Found: C, 41.93; H, 5.05; N, 7.73\%. ${ }^{1} \mathrm{H}-\mathrm{NMR}\left(\mathrm{CD}_{2} \mathrm{Cl}_{2}\right): \delta 1.52\left(\mathrm{~m}, 12 \mathrm{H}, \mathrm{CH}_{3} \mathrm{CH}_{2}\right)$, $3.96\left(\mathrm{~m}, 8 \mathrm{H}, \mathrm{CH}_{3} \mathrm{CH}_{2}\right), 7.64(\mathrm{~m}, 5 \mathrm{H}, \mathrm{Ph}), 7.73(\mathrm{~d}, 1 \mathrm{H}$, $\left.\mathrm{CNCH}_{2} \mathrm{Ph},{ }^{2} J_{\mathrm{H}, \mathrm{H}}=7.2 \mathrm{~Hz}\right), 7.77\left(\mathrm{~d}, 1 \mathrm{H}, \mathrm{CNCH}_{2} \mathrm{Ph}\right.$, $\left.{ }^{2} J_{\mathrm{H}, \mathrm{H}}=7.2 \mathrm{~Hz}\right)$. IR $\left(\mathrm{CH}_{2} \mathrm{Cl}_{2}, \mathrm{~cm}^{-1}\right): v_{\mathrm{CN}}, 2105(\mathrm{~s}) ; v_{\mathrm{CO}}$, 1958 (s). IR (KBr, $\left.\mathrm{cm}^{-1}\right)$ : $v_{\mathrm{CN}}, 2089(\mathrm{~s}) ; v_{\mathrm{CO}}, 1938$ (s).

\subsection{X-ray data collection, solution and refinement}

Data were collected at $150 \mathrm{~K}$ in a Siemens SMARTCCD instrument, equipped with a normal focus and 3 $\mathrm{kW}$ sealed-tube X-ray source. The structures of $\mathbf{1}$ and $\mathbf{5}$ were solved by heavy-atom methods and refined by a full-matrix least-squares procedure using NRCVAX [6]. All the non-hydrogen atoms were refined anisotropically. The other essential details of single-crystal data 
measurement and refinement are given in Table 1. One chlorine atom of $\mathrm{CH}_{2} \mathrm{Cl}_{2}$ in the asymmetric unit of the crystal of $\mathbf{5}$ is disordered and two chlorine positions with 70 and $30 \%$ occupancy were assigned to $\mathrm{Cl}(1)$ and $\mathrm{Cl}\left(1^{\prime}\right)$, respectively. There is a residual peak with 2.040 $\mathrm{e} \AA^{-3}$ in a distance of $0.09 \AA$ close to the $\mathrm{Cl}(1)$ atom on the last difference Fourier map.

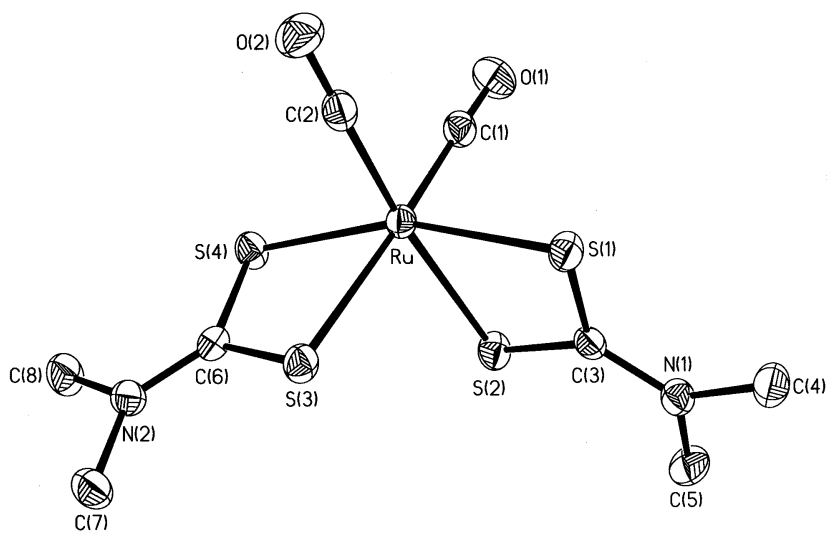

Fig. 1. ORTEP plot of 1 with $50 \%$ thermal ellipsoids. Selected bond lengths: $\quad \mathrm{Ru}-\mathrm{C}(1)=1.887(3), \quad \mathrm{Ru}-\mathrm{C}(2)=1.885(3), \quad \mathrm{Ru}-\mathrm{S}(1)=$ 2.4146(7), $\quad \mathrm{Ru}-\mathrm{S}(2)=2.4586(7), \quad \mathrm{Ru}-\mathrm{S}(3)=2.4566(7), \quad \mathrm{Ru}-\mathrm{S}(4)=$ $2.4144(7), \quad \mathrm{S}(1)-\mathrm{C}(3)=1.726(3), \quad \mathrm{S}(2)-\mathrm{C}(3)=1.724(3), \quad \mathrm{S}(3)-\mathrm{C}(6)=$ 1.730(3), $\quad \mathrm{S}(4)-\mathrm{C}(6)=1.731(3), \quad \mathrm{C}(1)-\mathrm{O}(1)=1.144(3), \quad \mathrm{C}(2)-\mathrm{O}(2)=$ 1.151(3), $\mathrm{N}(1)-\mathrm{C}(3)=1.328(3), \quad \mathrm{N}(2)-\mathrm{C}(6)=1.325(3) \quad \AA$. Selected bond angles: $\mathrm{C}(1)-\mathrm{Ru}-\mathrm{C}(2)=91.90(11), \mathrm{C}(1)-\mathrm{Ru}-\mathrm{S}(1)=93.44(8)$, $\mathrm{S}(1)-\mathrm{Ru}-\mathrm{S}(2)=72.46(2), \quad \mathrm{S}(1)-\mathrm{Ru}-\mathrm{S}(3)=93.67(2), \quad \mathrm{S}(1)-\mathrm{Ru}-\mathrm{S}(4)=$ 161.86(2), $\quad \mathrm{S}(2)-\mathrm{Ru}-\mathrm{S}(3)=89.03(2), \quad \mathrm{S}(2)-\mathrm{Ru}-\mathrm{S}(4)=95.11(2)$, $\mathrm{S}(3)-\mathrm{Ru}-\mathrm{S}(4)=72.49(2), \mathrm{C}(2)-\mathrm{Ru}-\mathrm{S}(4)=93.17(8)^{\circ}$.

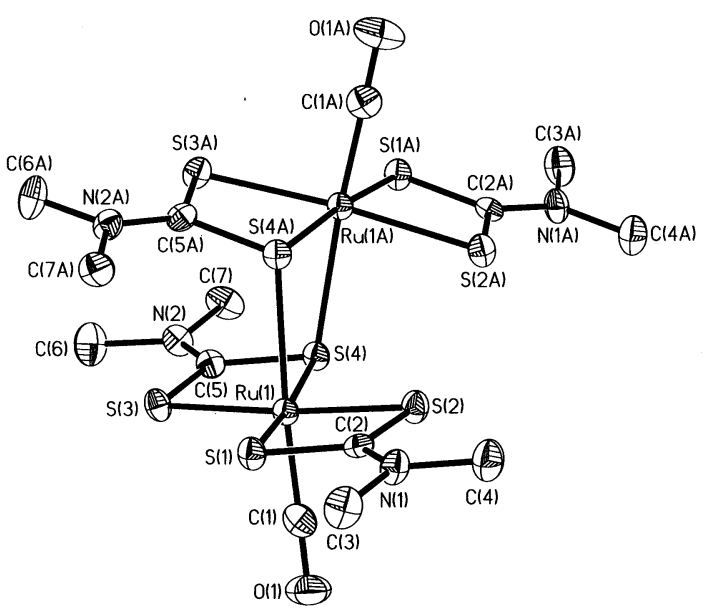

Fig. 2. ORTEP plot of 5 with $50 \%$ thermal ellipsoids. Selected bond lengths: $\mathrm{Ru}(1)-\mathrm{C}(1)=1.814(7), \mathrm{Ru}(1)-\mathrm{S}(1)=2.395(2), \mathrm{Ru}(1)-\mathrm{S}(2)=$ 2.412(2), $\quad \mathrm{Ru}(1)-\mathrm{S}(3)=2.399(2), \quad \mathrm{Ru}(1)-\mathrm{S}(4)=2.410(2), \quad \mathrm{Ru}(1)-$ $\mathrm{S}(4 \mathrm{~A})=2.554(2), \quad \mathrm{S}(1)-\mathrm{C}(2)=1.721(7), \quad \mathrm{S}(2)-\mathrm{C}(2)=1.731(6), \quad \mathrm{S}(3)-$ $\mathrm{C}(5)=1.701(7), \quad \mathrm{S}(4)-\mathrm{C}(5)=1.772(7), \quad \mathrm{C}(1)-\mathrm{O}(1)=1.165(9)$, $\mathrm{N}(1)-\mathrm{C}(2)=1.319(8), \mathrm{N}(2)-\mathrm{C}(5)=1.324(8) \AA$. Selected bond angles: $\mathrm{C}(1)-\mathrm{Ru}(1)-\mathrm{S}(1)=90.5(2), \quad \mathrm{S}(1)-\mathrm{Ru}(1)-\mathrm{S}(2)=72.81(6), \quad \mathrm{S}(1)-\mathrm{Ru}(1)-$ $\mathrm{S}(3)=106.75(6), \quad \mathrm{S}(3)-\mathrm{Ru}(1)-\mathrm{S}(4)=73.30(6), \quad \mathrm{S}(2)-\mathrm{Ru}(1)-\mathrm{S}(4)=$ 106.62(6), $\quad \mathrm{S}(4)-\mathrm{Ru}(1)-\mathrm{S}(4 \mathrm{~A})=83.16(6), \quad \mathrm{Ru}(1)-\mathrm{S}(4)-\mathrm{Ru}(1 \mathrm{~A})=$ $95.41(6)^{\circ}$.

\section{Results and discussion}

Reactions of $\left[\mathrm{Ru}_{2}(\mathrm{CO})_{4}\left(\mathrm{MeCN}_{6}\right]_{6}\left[\mathrm{BF}_{4}\right]_{2}\right.$ or $\left[\mathrm{Ru}_{2}(\mathrm{CO})_{4}\left(\mu-\mathrm{O}_{2} \mathrm{CMe}\right)_{2}(\mathrm{MeCN})_{2}\right]$ with uninegative 1,1 dithiolate anions, $(\mathrm{S}, \mathrm{S})^{-}$, occur as expected [1] to produce a series of mononuclear products, cis $-\left[\mathrm{Ru}(\mathrm{CO})_{2}-\right.$ $\left.\left(\eta^{2}-(\mathrm{SS})\right)_{2}\right]\left((\mathrm{SS})^{-}=\mathrm{Et}_{2} \mathrm{NCS}_{2}^{-}\right.$(2), ${ }^{t} \mathrm{BuSCS}_{2}^{-}$(3), and $(\mathrm{EtO})_{2} \mathrm{PS}_{2}^{-}$(4)). However, the reaction with potassium dimethyldithiocarbamate affords a mixture of two products, $\quad c i s-\left[\mathrm{Ru}(\mathrm{CO})_{2}\left(\eta^{2}-\mathrm{Me}_{2} \mathrm{NCS}_{2}\right)_{2}\right] \quad$ (1) and $\left[\mathrm{Ru}(\mathrm{CO})\left(\eta^{2}-\mathrm{Me}_{2} \mathrm{NCS}_{2}\right)\left(\mu, \eta^{2}-\mathrm{Me}_{2} \mathrm{NCS}_{2}\right)\right]_{2}(5)$, which are difficult to separate. Fortunately, after several attempts, satisfactory separation of the two products was finally achieved by the tedious fractional crystallization method. A ${ }^{1} \mathrm{H}-\mathrm{NMR}$ spectrum of the reaction solution after complete conversion indicated that the ratio between $\mathbf{1}$ and $\mathbf{5}$ is 3.35 and 4.63, respectively, favoring $\mathbf{1}$. Apparently the lightly stabilized $\mathrm{MeCN}$ ligands of $\left[\mathrm{Ru}_{2}(\mathrm{CO})_{4}(\mathrm{MeCN})_{6}\right]\left[\mathrm{BF}_{4}\right]_{2}$ are replaced more readily than the bound acetate ligands of $\left[\mathrm{Ru}_{2}(\mathrm{CO})_{4}(\mu-\right.$ $\left.\mathrm{O}_{2} \mathrm{CMe}\right)_{2}(\mathrm{MeCN})_{2}$ ] by dithiolates to produce cis$\left[\mathrm{Ru}(\mathrm{CO})_{2}\left(\eta^{2}-(\mathrm{SS})\right)_{2}\right]$ with less selectivity.

Compounds 1 [7] and 2 [8] were reported earlier, but the preparation procedures, either via the direct substitution of cis-[ $\left.\mathrm{RuCl}_{2}(\mathrm{CO})_{2}\left(\mathrm{PPh}_{3}\right)_{2}\right]$ with $\mathrm{NaS}_{2} \mathrm{CNMe}_{2}$. $2 \mathrm{H}_{2} \mathrm{O}$ or via the oxidative addition of $\left[\mathrm{Ru}_{3}(\mathrm{CO})_{12}\right]$ with $\mathrm{R}_{2} \mathrm{NC}(\mathrm{S}) \mathrm{SSC}(\mathrm{S}) \mathrm{NR}_{2}(\mathrm{R}=\mathrm{Me}, \mathrm{Et})$, gave lower yields of the complexes, compared with that of ours. Like cis$\left[\mathrm{Ru}(\mathrm{CO})_{2}\left(\eta^{2}-\mathrm{S}_{2} \mathrm{PMe}_{2}\right)_{2}\right]$ [1], compounds 1-4 display two carbonyl stretching bands with almost equal intensity in the IR spectra measured either in $\mathrm{CH}_{2} \mathrm{Cl}_{2}$ or as a $\mathrm{KBr}$ disc, indicating that the two carbonyls are cis to each other in solution or in the solid state. Indeed, this feature is shown clearly in the solid-state structure of $\mathbf{1}$ (Fig. 1). The $\mathrm{C}-\mathrm{N}$ distances of 1.328(3) and 1.325(3) $\AA$ is indicative of the presence of a partial $\mathrm{C}=\mathrm{N}$ bond [9], which is found compatible with the two methyl signals observed in the ${ }^{1} \mathrm{H}-\mathrm{NMR}$ spectrum of $\mathbf{1}$ or 2 . The coordination environment of the metal with two mutually $c i s$ dithiolates is approximately octahedral with the angle, formed by two carbonyls, $\mathrm{C}(1) \mathrm{O}(1)$ and $\mathrm{C}(2) \mathrm{O}(2)$, as $91.90(11)^{\circ}$. Two short and two long $\mathrm{Ru}-\mathrm{S}$ bonds were found $(d(\mathrm{Ru}-\mathrm{S}(1))=2.4146(7)$ and $d(\mathrm{Ru}-\mathrm{S}(4))=2.4144(7) \quad$ versus $d(\mathrm{Ru}-\mathrm{S}(2))=2.4586(7)$ and $d(\mathrm{Ru}-\mathrm{S}(3))=2.4566(7) \AA)$. Since the two long bonds are trans to carbonyls, the lengthening is understandable in terms of trans influence of the carbonyl group. However, we cannot exclude the possible involvement of the steric repulsive interactions between the dithiolate groups.

The crystal structure of $\mathbf{5}$ was also determined by $\mathrm{X}$-ray diffraction methods to reveal the dimeric nature with two chelating-bridging dithiolates (Fig. 2). The molecule $\mathrm{CH}_{2} \mathrm{Cl}_{2}$ was found as the solvent of crystallization. There is a crystallographically imposed $C_{2}$ axis through the center of the plane defined by $\mathrm{Ru}(1)$, 
$\mathrm{Ru}(1 \mathrm{~A}), \mathrm{S}(4)$, and $\mathrm{S}(4 \mathrm{~A})$. Hence, the structure can be described as consisting of two $\left[\mathrm{Ru}(\mathrm{CO})\left(\eta^{2}-(\mathrm{SS})\right)_{2}\right]$ fragments. It is noteworthy that the relative orientation between the two chelating dithiolates in each fragment of $\mathbf{5}$ is trans, rather than cis as observed in $\mathbf{1}$. Hence, the structure of $\mathbf{5}$ adopts configuration $\mathbf{B}$. This structure is kept in solution as reflected in the IR and ${ }^{1} \mathrm{H}-\mathrm{NMR}$ spectra; only one carbonyl stretching band was observed in solution or in the solid state, and four methyl ${ }^{1} \mathrm{H}$ signals were observed in $\mathrm{CDCl}_{3}$. One sulfur atom, $\mathrm{S}(4)$ or $\mathrm{S}(4 \mathrm{~A})$, of one dithiolate group in each fragment acts as the bridging atom to connect with the metal atom in the other fragment in a position trans to the carbonyl group. The bridging $\mathrm{Ru}-\mathrm{S}$ bonding is apparently weaker with $d(\mathrm{Ru}(1)-\mathrm{S}(4 \mathrm{~A}))=2.554(2) \AA$ in $\mathbf{5}$, compared with the distances of 2.4586(7) and 2.4566(7) $\AA$ for similar bonds found in 1. Two such weak bridging bonding interactions in $\mathbf{5}$ is probably caused by the nonbonded repulsive interactions between the dithiolate groups in different fragment. As a result, the $\mathrm{C}-\mathrm{N}$ distances of $1.319(8)$ and $1.324(8) \AA$ in $\mathbf{5}$ are not significantly different from those in $\mathbf{1}$.

Compound $\mathbf{5}$ can be prepared alternatively via decarbonylation of $\mathbf{1}$, using trimethylamine $N$-oxide in $\mathrm{MeCN}$. However, the dimer $\left[\mathrm{Ru}(\mathrm{CO})\left(\eta^{2}-\right.\right.$ $\left.\left.\mathrm{Et}_{2} \mathrm{NCS}_{2}\right)\left(\mu, \eta^{2}-\mathrm{Et}_{2} \mathrm{NCS}_{2}\right)\right]_{2}(\mathbf{6})$, prepared from a similar decarbonylation reaction of 2 with $\mathrm{Me}_{3} \mathrm{NO}$, has a structure different from 5. Rather complicated ${ }^{1} \mathrm{H}-\mathrm{NMR}$ signals were observed with three resolved multiplets in an integration ratio of 9:9:6 at $\delta 1.19,1.24$, and 1.37, respectively. Apparently there are more than four methyl signals observed for $\mathbf{6}$. Though this compound displays in the IR spectrum one broad and strong carbonyl stretching band at $1925 \mathrm{~cm}^{-1}$ in $\mathrm{CH}_{2} \mathrm{Cl}_{2}$, it shows two sharp such bands at 1943 and $1924 \mathrm{~cm}^{-1}$ with almost equal intensity in the solid state. The broad band at $1925 \mathrm{~cm}^{-1}$ is probably caused by the overlapping of two bands at a close wave number. All these spectral evidences may suggest another configuration for $\mathbf{6}$, probably configuration C. However, both $\mathbf{5}$ and $\mathbf{6}$ reacts readily at ambient temperature with benzyl isocyanide to yield a product with a similar geometry, cis$\left[\mathrm{Ru}(\mathrm{CO})\left(\mathrm{CNCH}_{2} \mathrm{Ph}\right)\left(\eta^{2}-(\mathrm{SS})\right)_{2}\right]\left((\mathrm{SS})^{-}=\mathrm{Me}_{2} \mathrm{NCS}_{2}^{-}\right.$(7) and $\mathrm{Et}_{2} \mathrm{NCS}_{2}^{-}(\mathbf{8})$ ). The cis assignment is based on one set of the $\mathbf{A B}$ quartet observed in the ${ }^{1} \mathrm{H}-\mathrm{NMR}$ spectrum assigned to the benzyl hydrogen nuclei for $\mathbf{7}$ or $\mathbf{8}$.

Various kinds of structure for mono- and dinuclear compounds can be explained in terms of both electronic and steric factors. The steric repulsive interactions are believed to be present between any two cis chelating dithiolates in either mono- or dinulcear complexes. However, two strong $\pi$-acceptor ligands in all mononuclear species (i.e. two COs in 1-4 and one $\mathrm{CO}$ and one $\mathrm{RNC}$ in 7-8) prefer to locate at cis positions on an octahedral coordination sphere around the $\mathrm{Ru}$ atom to help releasing the accumulated charge density of the $\mathrm{Ru}$ complexes of two strong $\sigma$-donor ligands, dithiolates, via back donation. The electronic factor, rather than the steric factor, determines the cis-geometry as observed in 1-4 and 7-8. Thus, once the decarbonylation of cis-[Ru(CO) $\left.)_{2}\left(\eta^{2}-(\mathrm{SS})\right)_{2}\right](\mathbf{1}$ and $\mathbf{2})$ occurred in $\mathrm{MeCN}$ and the resulting intermediate $c i s-\left[\mathrm{Ru}(\mathrm{CO})(\mathrm{NCMe})\left(\eta^{2}-\right.\right.$ $(\mathrm{SS}))_{2}$ ] (9) with only one $\pi$-acceptor, $\mathrm{CO}$, formed, this intermediate isomerizes to relieve the steric congestion between the two cis chelating dithiolates and form trans $-\left[\mathrm{Ru}(\mathrm{CO})(\mathrm{NCMe})\left(\eta^{2}-(\mathrm{SS})\right)_{2}\right](\mathbf{1 0})$. The subsequent replacement of $\mathrm{MeCN}$ of $\mathbf{1 0}$ with a strong donating dithiolate of another species, $\mathbf{1 0}$ or $\mathbf{9}$, forms a dimer $\left[\mathrm{Ru}(\mathrm{CO})\left(\eta^{2}-(\mathrm{S}, \mathrm{S})\right)\left(\mu, \eta^{2}-(\mathrm{S}, \mathrm{S})\right)\right]_{2}\left((\mathrm{SS})^{-}=\mathrm{Me}_{2} \mathrm{NCS}_{2}^{-}\right.$(5) or $\mathrm{Et}_{2} \mathrm{NCS}_{2}^{-}$(6)) in configuration $\mathbf{B}$ or $\mathbf{C}$, respectively (Scheme 1). The mononuclear intermediate trans$\left[\mathrm{Ru}(\mathrm{CO})\left(\mathrm{CNCH}_{2} \mathrm{Ph}\right)\left(\eta^{2}-(\mathrm{SS})\right)_{2}\right](\mathbf{1 1})$, if produced during the reaction of $\mathbf{5}$ and $\mathbf{6}$ with benzyl isocyanide in $\mathrm{CH}_{2} \mathrm{Cl}_{2}$, isomerize into cis- $\left[\mathrm{Ru}(\mathrm{CO})\left(\mathrm{CNCH}_{2} \mathrm{Ph}\right)\left(\eta^{2}-\right.\right.$ $\left.(\mathrm{SS}))_{2}\right]\left((\mathrm{SS})^{-}=\mathrm{Me}_{2} \mathrm{NCS}_{2}^{-}\right.$(7), $\left.\mathrm{Et}_{2} \mathrm{NCS}_{2}^{-}(\mathbf{8})\right)$. It is apparent that $\mathbf{1 1}$ is a kinetic product while $\mathbf{7}$ and $\mathbf{8}$ are thermodynamic products. No dinuclear products in configuration A was observed, probably indicating that the steric repulsive interactions between the two mutually $c$ is dithiolates in $c i s-\left[\mathrm{Ru}(\mathrm{CO})(\mathrm{NCMe})\left(\eta^{2}-(\mathrm{SS})\right)_{2}\right]$ are slightly larger than that in $c i s-\left[\mathrm{Ru}(\mathrm{CO})_{2}\left(\eta^{2}-(\mathrm{SS})\right)_{2}\right]$. Such repulsion may be increased appreciably during the dimerization, thus weakening the dimerization of cis$\left[\mathrm{Ru}(\mathrm{CO})(\mathrm{NCMe})\left(\eta^{2}-(\mathrm{SS})\right)_{2}\right] \quad$ into $\quad\left[\mathrm{Ru}(\mathrm{CO})\left(\eta^{2}-\right.\right.$ $\left.(\mathrm{S}, \mathrm{S}))\left(\mu, \eta^{2}-(\mathrm{S}, \mathrm{S})\right)\right]_{2}$ in configuration $\mathbf{A}$, a feature reminiscent of the $\mathbf{B}$ (back) strain in influencing the acid-base interaction [10].

\section{Conclusion}

The reaction of $\left[\mathrm{Ru}_{2}(\mathrm{CO})_{4}(\mathrm{MeCN})_{6}\right]\left[\mathrm{BF}_{4}\right]_{2}$ or $\left[\mathrm{Ru}_{2}(\mathrm{CO})_{4}\left(\mu-\mathrm{O}_{2} \mathrm{CMe}\right)_{2}(\mathrm{MeCN})_{2}\right]$ with potassium dimethyldithiocarbamate, sodium diethyldithiocarbamate, potassium tert-butylthioxanthate, and ammonium $O, O$ '-diethylthiophosphate gives both mono- and dimeric products of $c i s-\left[\mathrm{Ru}(\mathrm{CO})_{2}\left(\eta^{2}-(\mathrm{SS})\right)_{2}\right](\mathbf{1}-\mathbf{4})$ and $\left[\mathrm{Ru}(\mathrm{CO})\left(\eta^{2}-\mathrm{Me}_{2} \mathrm{NCS}_{2}\right)\left(\mu, \eta^{2}-\mathrm{Me}_{2} \mathrm{NCS}_{2}\right)\right]_{2}(\mathbf{5})$ with two structures 1 and $\mathbf{5}$ determined (Fig. 1 Fig. 2). The lightly stabilized $\mathrm{MeCN}$ ligands of $\left[\mathrm{Ru}_{2}(\mathrm{CO})_{4}(\mathrm{MeCN})_{6}\right]\left[\mathrm{BF}_{4}\right]_{2}$ are replaced more readily than the bound acetate ligands of $\left[\mathrm{Ru}_{2}(\mathrm{CO})_{4}(\mu-\right.$ $\left.\mathrm{O}_{2} \mathrm{CMe}\right)_{2}(\mathrm{MeCN})_{2}$ ] by dithiolates to produce cis$\left[\mathrm{Ru}(\mathrm{CO})_{2}\left(\eta^{2}-(\mathrm{SS})\right)_{2}\right]$ with less selectivity. Two dinuclear products 5 and $\mathbf{6}$ can also be prepared from the decarbonylation reaction of cis-[Ru(CO) $\left.\left.)_{2}\left(\eta^{2}-\mathrm{R}_{2} \mathrm{NCS}_{2}\right)\right]_{2}\right]$ $\left(\mathrm{R}=\mathrm{Me}(\mathbf{1})\right.$ and Et (2)) with $\mathrm{Me}_{3} \mathrm{NO}$. Although structure 5 adopts configuration $\mathrm{B}$, structure $\mathbf{6}$ takes configuration C. Both 5 and $\mathbf{6}$ react readily at ambient temperature with benzyl isocyanide to yield cis$\left[\mathrm{Ru}(\mathrm{CO})\left(\mathrm{CNCH}_{2} \mathrm{Ph}\right)\left(\eta^{2}-\mathrm{R}_{2} \mathrm{NCS}_{2}\right)_{2}\right](\mathrm{R}=\mathrm{Me}(7)$ and $\mathrm{Et}$ (8)). A dimerization pathway for cis- $\left[\mathrm{Ru}(\mathrm{CO})_{2}\left(\eta^{2}-\right.\right.$ 


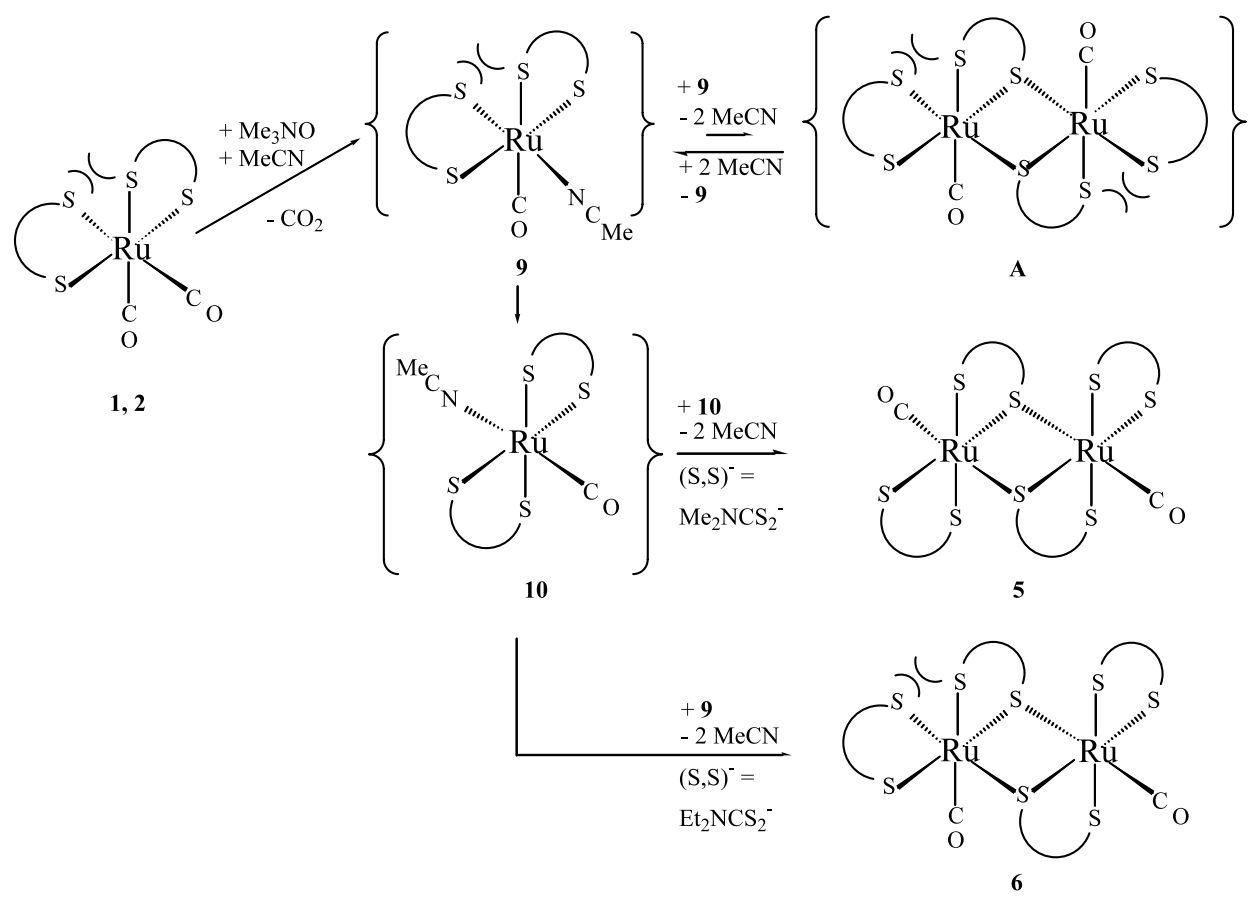

Scheme 1

$(\mathrm{SS}))_{2}$ ] via decabonylation and isomerization is proposed (Scheme 1).

\section{Supplementary material}

Crystallographic data for structural analysis have been deposited with the Cambridge Crystallographic Data Centre, CCDC nos. 173452 and 173453 for compounds $\mathbf{1}$ and $\mathbf{5}$, respectively. Copies of this information may be obtained free of charge from The Director, CCDC, 12 Union Road, Cambridge, CB2 1EZ, UK (Fax: +44-1223-336033; e-mail: deposit@ccdc. cam.ac.uk or www: http://www.ccdc.cam.ac.uk).

\section{Acknowledgements}

The authors thank the National Science Council of the Republic of China for financial support of this research (Contract No. NSC89-2113-M006-013).

\section{References}

[1] R.W. Hilts, M. Cowie, Inorg. Chem. 29 (1990) 3349.

[2] (a) K.-B. Shiu, C.-H. Li, T.-J. Chan, S.-M. Peng, M.-C. Cheng, S.-L. Wang, F.-L. Liao, M.Y. Chiang, Organometallics 14 (1995) 524

(b) K.-B. Shiu, L.-T. Yang, S.-W. Jean, C.-H. Li, R.-R. Wu, J.-C. Wang, L.-S. Liou, M.Y. Chiang, Inorg. Chem. 35 (1996) 7845 .

[3] J. Powell, M.J. Horvath, Organometallics 12 (1993) 4067.

[4] A. Author, in: F.A. Cotton, G. Wilkinson, C.A. Murillo, M. Bochmann (Eds.), Advanced Inorganic Chemistry, sixth ed., Wiley, New York, 1999, p. 541.

[5] G.R. Crooks, G. Gamlen, B.F.G. Johnson, J. Lewis, I.G. Williams, J. Chem. Soc. A (1969) 2761.

[6] E.J. Gabe, Y. Le page, J.-P. Charland, F.L. Lee, P.S. Lee, J. Appl. Crystallogr. 22 (1989) 384.

[7] D.J. Cole-Hamilton, T.A. Stephenson, J. Chem. Soc. Dalton Trans. (1974) 739.

[8] A.J. Deeming, R. Vaish, J. Organomet. Chem. 460 (1993) C8.

[9] K.-B. Shiu, S.-T. Lin, S.-M. Peng, M.-C. Cheng, Inorg. Chim. Acta 229 (1995) 153.

[10] H.C. Brown, J. Chem. Soc. (1956) 1248. 